Pacific

Journal of

Mathematics

FIRST ORDER DIFFERENTIAL OPERATORS ON A LOCALLY SYMMETRIC SPACE

H.D. Fegan and B. Steer 


\title{
FIRST ORDER DIFFERENTIAL OPERATORS ON A LOCALLY SYMMETRIC SPACE
}

\author{
H.D. FEgAN AND B. STEER
}

\begin{abstract}
The basic result is the classification of first order invariant elliptic differential operators on a quotient of a spin symmetric space by a suitable discrete group: Such operators are all twisted Dirac operators. As a consequence we obtain conditions for the spectral symmetry to be equivariant. We also show that the characteristic numbers of these spaces vanish, a result previously obtained by Hirzebruch and Slodowy from the study of elliptic genera.
\end{abstract}

\section{Introduction.}

Here we prove that any first order invariant elliptic operator on a space $\Gamma \backslash M$, where $M=G / H$ is a spin symmetric space and $\Gamma \subset G$ is a discrete subgroup, is a twisted Dirac operator. Furthermore, we study the spectral symmetry of such operators when $\Gamma$ is co-compact giving simple conditions for spectral symmetry and, if $G$ is compact, when this spectral symmetry is equivariant with respect to $G$.

A symmetric space is given as $M=G / H$ where $G$ and $H$ are Lie groups such that there is an involution $\theta$ on the Lie algebra, $\mathfrak{g}$, of $G$ with fixed set the Lie algebra $\mathfrak{h}$ of $H$; and now $M$ is compact if and only if $G$ is compact. (Since every compact group has a noncompact dual we see that every compact symmetric space has a noncompact dual, and conversely.) When $G$ is compact we may take $\Gamma=\{1\}$ and if $G$ is not compact there are always co-compact subgroups $\Gamma$, see $[\mathbf{9}]$.

Given a self-adjoint elliptic operator on a compact Riemannian manifold, there is a complete set of eigenvalues with finite dimensional eigenspaces. The eta function is defined as

$$
\eta(s)=\sum_{\lambda} \operatorname{sign}(\lambda)|\lambda|^{-s}, \quad \text { for } \operatorname{Re}(s) \gg 0,
$$

where the sum is over the nonzero eigenvalues $\lambda$ with each repeated as often as the dimension of the corresponding eigenspace. The eta invariant, defined by analytic continuation, is $\eta(0)$. This can be thought of as a second order invariant related to the $\hat{A}$-genus. For the $\hat{A}$-genus there is the AtiyahHirzebruch vanishing theorem when there is an $S^{1}$-action [3]. No such result 
can be expected for $\eta(0)$, as [15] shows, but it is known [8] that for a suitable action of $S^{1} \times S^{1}$ there is vanishing for a 'split' Dirac operator. Here we show the strong result that $\eta(s)$ vanishes identically for symmetric spaces $G / H$ where rank $G$-rank $H \geq 2$. In [10] Hirzebruch and Slodowy show that the characteristic numbers of $G / H$ vanish if rank $H<\operatorname{rank} G$. (They show very much more.) We can deduce this result as a consequence of ours upon considering the product $G / H \times S^{3} / \Gamma$ where $\Gamma$ is a suitable subgroup of $S^{3}$. Eventually we show that such a result is a relatively simple algebraic one, which is rather different from the subtle ones like the original result of Atiyah-Hirzebruch and those concerning the vanishing of the elliptic genus. This is discussed in the appendix: the example of $S U(2)$. If a group acts on $M$ and the operator is invariant then there is an induced action on each eigenspace. This gives rise to an equivariant version of both the eta function and the eta invariant. Both depend upon the metric and have been much studied, see [4] and [13]. Spectral symmetry, and especially equivariant symmetry, imply $\eta(s)=0$ and so show very strongly the vanishing of the eta invariant. In the case of certain locally symmetric spaces of rank 1 the eta function can be related to the geometry of $\Gamma,[\mathbf{1 2}]$ and $[\mathbf{1 5}]$.

Our results can be summarized as follows. In all cases $M=G / H$ is a spin symmetric space of either compact or noncompact type and $\Gamma \subset G$ is a co-compact discrete subgroup.

Theorem 1.1. If $M$ is compact and $D$ is a twisted Dirac operator on a homogeneous bundle over $M$, then $D$ has spectral symmetry and this symmetry is equivariant for the action of $H$.

For equivariant symmetry:

Theorem 1.2. If $D$ is a twisted Dirac operator on $\Gamma \backslash M$ then there are two cases:

(i) If rank $G$-rank $H \leq 1 D$ does not have $G$-equivariant spectral symmetry.

(ii) If rank $G$-rank $H \geq 2 D$ has $G$-equivariant spectral symmetry.

The classification is given by:

Theorem 1.3. If $D$ is a first order elliptic invariant operator on $M$, then $D$ is a twisted Dirac operator plus a bundle map.

Compare this to the results given in [16].

One aspect of Theorem 1.2 is that it divides symmetric spaces into three classes. If rank $G=\operatorname{rank} H$ then, for the weight $\mu$, index $D_{\mu}^{+}=d\left(\mu-\rho_{n}\right)$ where $d(\alpha)$ is the dimension of the representation with highest weight $\alpha$. So here there is very definite non-vanishing. When $\operatorname{rank} G \geq \operatorname{rank} H+2$ there is $G$-equivariant vanishing of the eta function if the dimension is odd (and of the index if $n$ is even). When $\operatorname{rank} G=\operatorname{rank} H+1$ this does not hold, 
although it is possible that the eta invariant could vanish, but it does not do so equivariantly.

The lack of symmetry for a first order invariant operator on $\Gamma \backslash M$ represents a secondary invariant of $\Gamma$. Since any such operator is a twisted Dirac operator plus a bundle map, our results show that for operators with no constant term all these secondary invariants vanish.

Section 2 establishes the basic definitions. Section 3 gives the proof of Theorem 1.1. In Section 4 we prove Theorem 1.2 in the case when $\Gamma=\{1\}$ and $M$ is compact. For $G$-equivariant symmetry passing from $\Gamma=\{1\}$ to any $\Gamma$ is automatic. Section 5 gives the passage between the compact and noncompact duals and the classification, Theorem 1.3, is obtained in Section 6. In Section 7 we give an application of these results to the classification of first order elliptic operators on a compact Riemannian spin manifold. If the operator acts invariantly on sections of a bundle associated with the frame bundle by a representation, then the operator is a twisted Dirac operator plus a bundle map. Finally, there are two appendices: The first describes the case of $S U(2)$ and the second discusses the difference rank of $M=\operatorname{rank}$ $G$-rank $H$, which is not the same as the rank of $M$.

Both authors thank the referee for his suggestions and in particular for pointing out that a result of $[\mathbf{1 0}]$ follows from one of theirs.

The first author was supported by the C.C. Hsiung fund.

\section{The Dirac Operator on a Symmetric Space.}

Let $M=G / H$ be a symmetric space of compact or non-compact type. The Lie algebra of $G$ then decomposes: $\mathfrak{g}=\mathfrak{m} \oplus \mathfrak{h}$; and the adjoint action of $G$ restricts to $A d: H \rightarrow S O(\mathfrak{m})$. We suppose that this lifts to spin: $\widetilde{A d}: H \rightarrow$ $\operatorname{Spin}(\mathfrak{m})$. If not, then there is a cover $H_{1}$ of $H$, which is either trivial or twofold, such that $H_{1}$ has an adjoint action which does lift and we can replace $G$ and $H$ by suitable double covers if necessary, see [14, §2]. If $G$ is compact, using the negative of the Killing form to obtain a bi-invariant metric on $\mathfrak{g}$, choose an orthonormal basis $\left\{E_{1}, \ldots, E_{m+h}\right\}$ for $\mathfrak{g}$ such that $\left\{E_{\alpha}: 1 \leq \alpha \leq m\right\}$ is a basis for $\mathfrak{m}$ and $\left\{E_{i}: m+1 \leq i \leq m+h\right\}$ is a basis for $\mathfrak{h}$. In the non-compact case, take the negative of the Killing form on $\mathfrak{h}$. Unless otherwise stated, we shall use Greek letters for indices in the range 1 to $m$ and Latin for $m+1$ to $m+h$. Further, let $p_{0}=1 H$ be the element of $M$ which is the image of the identity element of $G$.

We construct the usual local vector fields on $M$, see [9, Ch. IV Lemma 3.2]. Pick a subset $B \subset G$ such that $\pi: B \rightarrow B_{r}\left(p_{0}\right)$ homeomorphically onto an open geodesic ball in $M$. There is an open subset $U \subset H$ containing the identity so that $B U$ is an open neighbourhood of the identity in $G$. The vector fields are then $E_{\alpha}$ transferred from $\mathfrak{m}$ to $M$ and are invariant under 
translation by $B$. Using the homogeneous structure we define coordinates on $B_{r}(p)$ for each $p \in M$.

Since $M$ is a symmetric space, the decomposition $\mathfrak{g}=\mathfrak{m} \oplus \mathfrak{h}$ has the properties $[\mathfrak{h}, \mathfrak{h}] \subset \mathfrak{h},[\mathfrak{h}, \mathfrak{m}] \subset \mathfrak{m}$ and $[\mathfrak{m}, \mathfrak{m}] \subset \mathfrak{h}$. In the compact case, the connection on $G$ associated to the bi-invariant metric is $\nabla_{X} Y=\frac{1}{2}[X, Y]$, for left invariant vector fields $X$ and $Y$. Transferring this connection to $M$ yields a connection such that $\nabla_{E_{\alpha}} E_{\beta}=0$ at $p_{0}$, since $\left[E_{\alpha}, E_{\beta}\right] \in \mathfrak{h}$ and so is zero in $M$. Observe that this is only true at one point. However, the formulae obtained are then true point by point. Similar formulae hold in the non-compact case and will be dealt with in Section 5 .

Since $M$ is spin symmetric we have a homogeneous spin bundle on $M$. Let $\Delta: \operatorname{Spin}(\mathfrak{m}) \rightarrow$ Aut $S$ be the spin representation then the spin representation of $H$ is $\chi=\Delta \circ \widetilde{A d}: H \rightarrow \operatorname{Spin}(\mathfrak{m}) \rightarrow$ Aut $S$. The spin bundle is $\mathbf{S}=G \times_{H} S$, where $H$ acts on $S$ by $\chi$ and on $G$ by right translation. Thus the sections of $\mathbf{S}$ decompose: $\Gamma(\mathbf{S})=\left(C^{\infty}(G) \otimes S\right)^{H}$ where the superscript $H$ indicates that we take the subspace of $C^{\infty}(G) \otimes S$ which is invariant under $H$.

More generally let $\pi: H \rightarrow$ Aut $V$ be a representation on a finite dimensional vector space $V$. Then construct the associated vector bundle $\mathbf{V}=G \times{ }_{H} V$ over $M=G / H$. Give $V$ an $H$-invariant inner product and let $\nabla^{V}$ be the Levi-Civita connection on $\mathbf{V}$. The spin bundle twisted by $V$ is then $\mathbf{S} \otimes \mathbf{V}$ which has the connection $\nabla=\nabla^{S} \otimes 1+1 \otimes \nabla^{V}$, where $\nabla^{S}$ is the spinor connection. The sections of $\mathbf{S} \otimes \mathbf{V}$ can again be identified as: $\Gamma(\mathbf{S} \otimes \mathbf{V})=\left(C^{\infty}(G) \otimes S \otimes V\right)^{H}$, where the $H$ superscript indicates that we take the $H$-invariant part.

Let $\left\{v_{1}, \ldots, v_{k}\right\}$ be a basis for $V$ then, under the action of $B$, we have locally a basis of sections for $\mathbf{V}$. Denote these local sections by $v_{1}, \ldots, v_{k}$. Since the right action by $H$ on $G$ commutes with the left action by $B$ on $G$ we have $\nabla_{E_{\alpha}}^{V} v_{i}=0$ for all $E_{\alpha}$ and $v_{i}$, that is, the sections of $\mathbf{V}$ defined locally by the action of $B$ are parallel along the normal coordinates.

The twisted Dirac operator is defined by $P: \Gamma(\mathbf{S} \otimes \mathbf{V}) \rightarrow \Gamma(\mathbf{S} \otimes \mathbf{V})$ is defined by $P=\sum_{\alpha} \omega E_{\alpha} \nabla_{E_{\alpha}}$. Here $E_{\alpha}$ acts by Clifford multiplication and $\omega$ is the volume form on $M$, normalized as to sign, so $\omega=i^{s} E_{1} \cdots E_{m}$ with $s=(m+1)(m+2) / 2$. The effect of this normalization is to give $\omega E_{\alpha} \omega=E_{\alpha}$ and $\omega^{2}=(-1)^{(m-1)}$. If $\operatorname{dim} \mathfrak{m}$ is even, the space of spinors splits $S=S^{+} \oplus S^{-}$and so $P=P^{+} \oplus P^{-}$. Now, because the connection on $M$ satisfies $\nabla_{E_{\alpha}} E_{\beta}=0$ at the point $p_{0}$, the spinor connection on a basic spinor $\psi=s \otimes 1$ (where $s \in S$ ) is given by $\nabla_{E_{\alpha}} \psi=0$. Thus, we have

$$
P(f \otimes s \otimes v)=\sum_{\alpha} E_{\alpha}(f) \otimes \omega E_{\alpha} s \otimes v .
$$

A direct calculation as in [14, Proposition 3.2], or the appendix of [5], using the fact that the induced action of $X \in \mathfrak{h}$ on $S$ is given by $\chi(X)=$ 
$-\frac{1}{4} \Sigma_{\alpha}\left[X, E_{\alpha}\right] E_{\alpha}$ yields $P^{2}=\left(\Omega_{G}-\Omega_{H}\right) \otimes 1 \otimes 1-2 \Sigma_{i} E_{i} \otimes \chi\left(E_{i}\right) \otimes 1, \Omega_{G}=$ $-\Sigma_{\alpha} E_{\alpha}^{2}-\Sigma_{i} E_{i}^{2}$ and $\Omega_{H}=-\Sigma_{i} E_{i}^{2}$. Now, since we are acting on the $H$ invariant elements of $C^{\infty}(G) \otimes S$, we get the following proposition:

Proposition 2.1. The square of the Dirac operator is $P^{2}=\Omega_{G} \otimes 1 \otimes 1+$ $1 \otimes \chi\left(\Omega_{H}\right) \otimes 1$.

Remark 2.2. The operators $\Omega_{G}$ and $\Omega_{H}$ are the Casimirs on $G$ and $H$. In principle, this gives the eigenvalues of $P^{2}$, and an explicit formula is given in [14, Proposition 3.2]. However, since we do not have the assumption rank $G=\operatorname{rank} H$, the representation structure of $\chi$ adds complication to the details.

For any operators $A$ and $B$, we define the commutator to be $[A, B]=$ $A B-B A$ and the anticommutator $\{A, B\}=A B+B A$. Let $E=i^{s} E_{1} \cdots E_{m-1}$ with $s=m(m-1) / 2$ and $F=i^{2 m-1} E_{m}$. This normalization has been chosen so that $E^{2}=1$. For any $E_{\alpha},\left[E_{\alpha}, F\right] \in \mathfrak{h}$ as elements of $\mathfrak{g}$. Thus, as vector fields on $M,\left[E_{\alpha}, F\right]=0$. Note that the operator $E$ is defined as an element in the Clifford algebra of $\mathfrak{g}$, while $F$ is in $\mathfrak{g}$. Then $E$ acts globally on $M$ by the spin structure while the action of $F$ is defined in each coordinate patch, $B_{r}(p)$, by right translation on $C^{\infty}(G)$. The relationship of these to the Dirac operator is given in the following two results:

Lemma 2.3. $\{P, E\}=2 F \otimes 1$ acting on $\left(C^{\infty}(G) \otimes S\right)^{H}$.

Proof. Here $E$ acts on $\left(C^{\infty}(G) \otimes S\right)^{H}$ by $1 \otimes E$. The result is now obtained, using $E=i^{2 m+1} \omega E_{m}$, by the following calculation:

$$
\begin{aligned}
\{P, E\} & =\sum_{\alpha} E_{\alpha} \otimes\left(\omega E_{\alpha} E+E \omega E_{\alpha}\right) \\
& =i^{2 m+1} \sum_{\alpha} E_{\alpha} \otimes\left(\omega E_{\alpha} \omega E_{m}+\omega E_{m} \omega E_{\alpha}\right) \\
& =i^{2 m+1} \sum_{\alpha} E_{\alpha} \otimes\left(E_{\alpha} E_{m}+E_{m} E_{\alpha}\right) \\
& =i^{2 m+1} E_{m} \otimes 2 E_{m}^{2} \\
& =2 F \otimes 1 .
\end{aligned}
$$

Lemma 2.4. $\left[P^{2}, E\right]=0$.

Proof. By Proposition $2.1 P^{2}=\Omega_{G} \otimes 1 \otimes 1+1 \otimes \chi\left(\Omega_{H}\right) \otimes 1$. Now $\Omega_{G} \otimes 1 \otimes 1$ commutes with $1 \otimes E \otimes 1$ and since $\Omega_{H}$ is in the centre of the enveloping algebra, $\chi\left(\Omega_{H}\right)$ commutes with $E$. Thus, $P^{2}$ commutes with $E$, obtaining the result. 


\section{The Cartan Involution and Spectral Symmetry.}

Using the Cartan involution we can easily obtain spectral symmetry for the Dirac operator when $G$ is compact and $A d$ lifts to spin. Let $\theta: G \rightarrow G$ be the Cartan involution. Then $\theta \mid H$ is the identity, and $\theta$ induces an inversion map, also denoted by $\theta$, on $M: \theta: M \rightarrow M$.

Lemma 3.1. The inversion map $\theta$ intertwines $P$ and $-P$, i.e., $P \theta=-\theta P$.

Proof. The derivative $d \theta: \mathfrak{m} \rightarrow \mathfrak{m}$ is multiplication by -1 . Thus, for the connection on $M$ :

$$
\nabla_{X} \circ \theta=\theta \circ \nabla_{d \theta(X)}=\theta \circ \nabla_{-X}=-\theta \circ \nabla_{X}
$$

Now if $X \in \mathfrak{m}$ acts on $S$, by Clifford multiplication we have $X \circ \theta(f \otimes s)=$ $\theta f \otimes X s=\theta \circ X(f \otimes s)$, on $\left(C^{\infty}(G) \otimes S\right)^{H}$. Since $P=\sum_{\alpha} \omega E_{\alpha} \nabla_{E_{\alpha}}$ the result is immediate.

Corollary 3.2. The Dirac operator has $H$-equivariant spectral symmetry.

Proof. If $\lambda$ is an eigenvalue of $P$ with eigenspace $V_{\lambda}$ then $-\lambda$ is also an eigenvalue with eigenspace $V_{-\lambda}$ then $-\lambda$ is also an eigenvalue with eigenspace $V_{-\lambda}=\theta V_{\lambda}$. Both eigenspaces are representation spaces for $G$ and by restriction for $H$. Since $\theta \mid H$ is the identity, $V_{\lambda}$ and $V_{-\lambda}$ are isomorphic as $H$ representation spaces.

Remark 3.3. Note that $\theta$ is not the identity map on $G$ and so the question of $G$-equivariance is not addressed by this result.

\section{The Space of Spinors and Symmetry in the Compact Case.}

Let $T_{G} \subset G$ be a maximal torus of $G$ such that $T_{H}=T_{G} \cap H$ is the maximal torus of $H$. We fix these tori and express weights of representation using them. Then we denote the positive roots of $G$ by $\alpha_{1}, \ldots, \alpha_{\mu}$, which are ordered so that $\widetilde{\alpha}_{i}=\alpha_{i} \mid T_{H}(1 \leq i \leq \nu)$ are the positive roots of $H$. Define $\rho=\frac{1}{2} \sum_{i=1}^{\mu} \widetilde{\alpha}_{i}, \rho_{k}=\frac{1}{2} \sum_{i=1}^{\nu} \widetilde{\alpha}_{i}$ and $\rho_{n}=\frac{1}{2} \sum_{i=\nu+1}^{\mu} \widetilde{\alpha}_{i}$ so $\rho=\rho_{k}+\rho_{n}$, where $\widetilde{\alpha}_{i}=\alpha_{i} \mid T_{H}$ for all $i$.

The decomposition $\mathfrak{g}=\mathfrak{h} \oplus \mathfrak{m}$ gives rise to an adjoint representation Ad $: H \rightarrow S O(\mathfrak{m})$ which, by taking double covers as explained earlier, we suppose lifts to spin: $\widetilde{A d}: H \rightarrow \operatorname{Spin}(\mathfrak{m})$. The spin representation is $\Delta: \operatorname{Spin}(\mathfrak{m}) \rightarrow \operatorname{Aut} S$, which defines $S$; see [1] or [2] for a more detailed description. Let $m=\operatorname{dim}(\mathfrak{m})$, then $\operatorname{dim} S=2^{k}, k=[\mathfrak{m} / 2]$, and $\chi=\Delta \circ \widetilde{A d}$. Then the weights of $\chi$ are given as follows: Let $\pm \lambda_{1}, \pm \lambda_{2}, \pm \lambda_{3}, \ldots, \pm \lambda_{k}$ be the nonzero weights of the standard representation of $S O(\mathfrak{m})$, ordered 
lexicographically. Then the weights of $\Delta$ are

$$
\frac{1}{2}\left( \pm \lambda_{1} \pm \lambda_{2} \cdots \pm \lambda_{k}\right) \text {. }
$$

If $m$ is odd $\Delta$ is irreducible; if $m$ is even $\Delta=\Delta^{+} \oplus \Delta^{-}$with the parity of the signs in (4.1) giving $\Delta^{+}$(even number of - ) and $\Delta^{-}$(odd number of $-)$. The weights of $\chi$ are then given by

$$
\frac{1}{2}\left( \pm \alpha_{\nu+1} \pm \cdots \pm \alpha_{\mu}\right) \text {. }
$$

These are obtained, as in $[\mathbf{1 4}, \S 2]$ from (4.1) by replacing $\lambda_{i}$ with $\alpha_{i}$ if $\nu+1 \leq i \leq \mu$ and all other $\lambda_{i}$ by zero. The multiplicity of a weight is given by the number of ways it occurs. Here $\alpha_{i}$ are the weights of $G$ ordered so the first $\nu$ restrict to give weights of $H$.

Now consider the root space decomposition of $\mathfrak{g}=\bigoplus_{i=1}^{\mu} \mathfrak{g}_{\alpha_{i}} \oplus t_{G}$ and set $t_{0}=t_{G} / t_{H}$. Then $\mathfrak{m}$ can be decomposed as:

$$
\mathfrak{m}=\bigoplus_{i=\nu+1}^{\mu} \mathfrak{g}_{\alpha_{i}} \oplus t_{0}=\mathfrak{p} \oplus t_{0} .
$$

Now $\operatorname{dim} \mathfrak{p}$ is even and let $r=\left[\frac{1}{2} \operatorname{dim} t_{0}\right]$ be the integer part of $\frac{1}{2} \operatorname{dim} t_{0}$. We write $S(\mathfrak{m})$ for the spinor space of $\mathfrak{m}$, replacing $S$, and $S(\mathfrak{p})$ for that of $\mathfrak{p}$.

Lemma 4.1. If $r=0$ then $V_{\rho_{n}}$, the representation of $H$ with highest weight $\rho_{n}$, is an irreducible component of $\chi$ with multiplicity one.

Proof. Since $r=0$ either $\operatorname{dim} t_{0}=0$ or $\operatorname{dim} t_{0}=1$.

If $\operatorname{dim} t_{0}=0$ then $\mathfrak{m}=\mathfrak{p}$ and from (4.2) it is immediate that $V_{\rho_{n}}$ occurs with multiplicity one.

If $\operatorname{dim} t_{0}=1$ then $S(\mathfrak{m})=S(\mathfrak{p})$ and these are equivalent as representations of $H$, although not as representations of the underlying spin groups. Again, the result of the lemma follows from (4.2).

Remark 4.2. If $r \geq 1$ then $\operatorname{dim} S(\mathfrak{m})=2^{r} \operatorname{dim} S(\mathfrak{p})$. Further, from (4.2), we see that $S(\mathfrak{m})=2^{r} S(\mathfrak{p})$ as representation spaces of $H$.

Using these results and the operators $E$ and $F$ of Section 2, we can now prove Theorems 1.1 and 1.2 for a compact symmetric space.

Theorem 4.3. If $\operatorname{dim} t_{0} \leq 1$ then $P$ does not have $G$-equivariant symmetry.

Proof. Suppose that $P$ does have $G$-equivariant symmetry. Using the PeterWeyl theorem as in $[6]\left(C^{\infty}(G) \otimes S\right)^{H}=\Sigma \tilde{V}_{\lambda} \otimes\left(\tilde{V}_{\lambda}^{*} \otimes S\right)^{H}$, so $\operatorname{dim}\left(\tilde{V}_{\lambda}^{*} \otimes\right.$ $S)^{H}$ must be even as there are equal multiplicities for positive and negative eigenvalues.

If $\lambda$ is a weight for $H$ which is also a weight for $G$, then we can form the irreducible representation $\tilde{V}_{\lambda}$ of $G$. As a representation of $H$ this is not 
necessarily irreducible and we decompose it: $\tilde{V}_{\lambda}=V_{\lambda} \oplus_{\mu} V_{\mu}$. The weights that occur as highest weights for these representations of $H$ must be weights of $\tilde{V}_{\lambda}$ as a representation of $G$. Thus $\lambda$ occurs with multiplicity one and if $\mu$ also occurs then $\lambda>\mu$ : Moreover $\mu$ must be a weight for $G$, though it may not be dominant. However, there will be a dominant weight for $G$ in the Weyl group orbit of $\mu$ and this will yield a representation of $H$.

Now consider the representation $\tilde{V}_{\rho_{n}}$ of $G$. This decomposes as a representation of $H: \tilde{V}_{\rho_{n}}=V_{\rho_{n}} \underset{\sim *}{\oplus} V_{\mu}$. By Lemma 4.1, $V_{\rho_{n}}$ occurs in $S$ with multiplicity one. Thus $\operatorname{dim}\left(\tilde{V}_{\rho_{n}}^{*} \otimes S\right)^{H}=1+\Sigma_{\mu} \operatorname{dim}\left(V_{\mu} \otimes S\right)^{H}$ and so, for some $\mu<\lambda, V_{\mu}$ occurs in $S$ with odd multiplicity. Without loss of generality, we may suppose $\mu$ is dominant and can repeat the argument with $\mu$ replacing $\rho_{n}$. By induction, we can find a sequence of weights $\mu_{i}$ such that $\mu_{i+1}<\mu_{i}$ and $V_{\mu_{i}}$ occurs in $S$ with odd multiplicity. This is clearly impossible, and so $P$ does not have $G$-equivariant symmetry.

Theorem 4.4. If $\operatorname{dim} t_{0} \geq 2$ then $P$ has $G$-equivariant symmetry.

Proof. Let $U_{p}$ be the subspace of $\operatorname{Cliff}(\mathfrak{m})$ spanned as a vector space by $E_{i_{1}} \cdots E_{i_{p}}, i_{1}<i_{2}<\cdots<i_{p}$. Then if $X \in U_{p}$ for $p \neq 0$ we have trace $X \mid S=0$. Thus we have

$$
\operatorname{trace} E \mid S=0
$$

Now using $\mathfrak{m}=\mathfrak{p} \oplus t_{0}$, let $\omega_{p}=E_{1} \cdots E_{p}$ where $\left\{E_{1}, \ldots, E_{p}\right\}$ is an orthonormal basis for $\mathfrak{p}$. Then $E=i^{s} \omega_{p} E_{p+1} \cdots E_{m-1}$ for $s=m(m-1) / 2$ and $E_{i} \in t_{0}, p+1 \leq i \leq m-1$. Now if $X \in t_{0}$ both $X$ and $\omega_{p}$ commute with the action of $H$ by $\chi$. Thus, $E$ is an $H$-map. Since $E^{2}=1$, decompose $S=S^{+} \oplus S^{-}$into +1 and -1 eigenspaces of $E$. Then $\operatorname{dim} S^{+}=\operatorname{dim} S^{-}$, since trace $E \mid S=0$. By Remark 4.2 we have $S=2^{r} S(\mathfrak{p})$ so, since $E$ is an $H$-map, we have

$$
S^{+} \cong S^{-} \cong 2^{r-1} S(\mathfrak{p})
$$

Decompose the sections $\mathbf{S}: \Gamma(\mathbf{S})=\oplus\left(\widetilde{V}_{\lambda} \otimes \widetilde{V}_{\lambda^{*}} \otimes S\right)^{H}=\oplus\left(\tilde{V}_{\lambda} \otimes\left(\tilde{V}_{\lambda *} \otimes\right.\right.$ $S)^{H}$ ) into isotypic components under the left action by $G$. By Proposition $2.1 P^{2}=\Omega_{G} \otimes 1 \otimes 1+1 \otimes 1 \otimes \Omega_{H}$, so each component is an invariant space for $P^{2}$ and, since $\Omega_{H}$ is constant on $S$ the eigenspaces of $P^{2}$ on $\left(\widetilde{V}_{\lambda} \otimes \widetilde{V}_{\lambda^{*}} \otimes S\right)^{H}$ are of the form $(W \otimes S)^{H}$. Now decompose $(W \otimes S)^{H}=\sum_{i}\left(W_{i} \otimes S\right)^{H}$ into eigenspaces of $F \otimes 1$. Then using (4.5) we get eigenspaces for $E$ :

$$
\left(W_{i} \otimes S\right)^{H}=\left(W_{i} \otimes S^{+}\right)^{H} \oplus\left(W_{i} \otimes S^{-}\right)^{H} .
$$


Relative to the decomposition (4.6) there are the block forms $E=\left(\begin{array}{cc}1 & 0 \\ 0 & -1\end{array}\right)$ and $P=\left(\begin{array}{ll}a & b \\ c & d\end{array}\right)$. A simple calculation shows

$$
\{P, E\}=\left(\begin{array}{cc}
2 a & 0 \\
0 & -2 d
\end{array}\right)=2 F \otimes 1,
$$

with the last equality from Lemma 2.3. Since $\left(W_{i} \oplus S\right)^{H}$ is an eigenspace for $F \otimes 1$, this is constant so $a=-d$. Now $(W \otimes S)^{H}$ is an eigenspace for $P^{2}$, so $P$ has two eigenvalues $\alpha$ and $-\alpha$ on $(W \otimes S)^{H}$. Since trace $P \mid\left(W_{i} \otimes S\right)^{H}=0$ we see that $P$ has spectral symmetry on $\left(W_{i} \otimes S\right)^{H}$. Thus $P$ has spectral symmetry on $\left(\widetilde{V}_{\lambda} \otimes \widetilde{V}_{\lambda^{*}} \otimes S\right)^{H}$. Now as a representation space of $G\left(\widetilde{V}_{\lambda} \otimes \widetilde{V}_{\lambda^{*}} \otimes S\right)^{H}=n \widetilde{V}_{\lambda}$ and, since the eigenspaces of $P$ are $G$ representation spaces, we see that each eigenspace of $P$ on $\left(\widetilde{V}_{\lambda} \otimes \widetilde{V}_{\lambda} \otimes S\right)^{H}$ is $n_{i} \widetilde{V}_{\lambda}$ as a representation space of $G$. Thus, the spectral symmetry of $P$ is $G$-equivariant symmetry.

We now consider the twisted Dirac operator. From (2.1) we have:

Lemma 4.5. Using normal coordinates $P_{V}=P \otimes 1$, where $P$ is the Dirac operator on $\mathbf{S}$ with no twisting.

Theorem 4.6. $\quad$ i. If $\operatorname{dim} t_{0}=1 P_{V}$ does not have G-equivariant spectral symmetry.

ii. If $\operatorname{dim} t_{0} \geq 2 P_{V}$ has $G$-equivariant spectral symmetry.

Proof. Use Lemma 4.5. Then the proof of Theorem 4.3 extends routinely to establish part i and the proof of Theorem 4.4 extends to give part ii.

Finally, we observe that the results of Section 3 using the Cartan involution also extend to the case of twisted Dirac operators. This establishes the following:

Theorem 4.7. The twisted Dirac operator has $H$-equivariant spectral symmetry in all cases.

\section{The Noncompact Case.}

Let $M=G / H$ be a symmetric space of noncompact type and $\Gamma \subset G$ be a discrete co-compact group, so $\Gamma \backslash M$ is compact. Then, we use the compact dual $G^{*}$ of $G$ to show spectral symmetry for the Dirac operator $P$.

The Lie algebra of $G$ decomposes:

$$
\mathfrak{g}=\mathfrak{m} \oplus \mathfrak{h} .
$$

Let $\mathfrak{g}_{\mathbb{C}}$ be the complexification of $\mathfrak{g}$, and $\mathfrak{g}^{*}$ be the compact dual $\mathfrak{g}^{*} \subset \mathfrak{g}_{\mathbb{C}}$. This has the following correspondence with $\mathfrak{g}$ :

$$
X \rightarrow X \text { for } x \in \mathfrak{h}, \quad X \rightarrow i X \text { for } X \in \mathfrak{m} .
$$


Denote by $X^{*}$ the element of $\mathfrak{g}^{*}$ corresponding to $X \in \mathfrak{g}$. There is then a correspondence between the Dirac operator $P$ on $M$ and its dual $P^{*}$ on $M^{*}=G^{*} / H$.

Lemma 5.1. $(P \psi)^{*}=P^{*} \psi^{*}$.

This follows directly from the definitions.

If $P_{V}$ is a twisted Dirac operator on $\Gamma \backslash M$, then using the correspondence of Lemma 5.1 yields the noncompact version of Theorem 4.6. This follows since the equivariance of the symmetry on $G / H$ means symmetry descends to $\Gamma \backslash G / H$.

Theorem 5.2. If rank $G$-rank $H \geq 2$ then $P_{V}$ has spectral symmetry.

Notice that $G$ does not act on $\Gamma \backslash G / H$, so we cannot discuss equivariant symmetry.

Finally we note that the result of Theorem 4.7 does not have a corresponding noncompact version. This result uses the inversion map $X \rightarrow X^{-1}$ induced by $G / H$. However, this does not necessarily pass to a map of spinors on $\Gamma \backslash M$; for example in the rank one case it fails. In the compact case we can take $\Gamma=\{1\}$ but this does not give a well-defined eta function in the noncompact case.

\section{First Order Invariant Elliptic Operators.}

Let $D$ be a first order invariant elliptic operator on a homogeneous bundle $\mathbf{E}$, with fibre $E$, over $M$. Then its symbol is a bilinear map:

$$
\sigma: \mathfrak{m} \otimes E \rightarrow E \text {. }
$$

This can be rewritten as $\sigma: \mathfrak{m} \rightarrow \operatorname{End}(E)$ and the condition that $D$ is elliptic is equivalent to $\sigma(x)$ is invertible if $x \neq 0$. Since $\mathbf{E}$ is a homogeneous bundle, $E$ is a complex vector space and, by a suitable choice of metric, we have $\sigma\left(S^{m-1}\right) \subset U(n)$ where $S^{m-1}$ is the unit sphere in $\mathfrak{m}$ and $U(n)$ is the unitary group for $n=\operatorname{dim} E$. By Lemma 4.1 of [7] we have:

Lemma 6.1. The symbol $\sigma$ induces an action $\phi$ of $\operatorname{spin}(\mathfrak{m})$ on $E$. Further, $\phi$ is the standard action, so $E=k S$ (if $m$ is odd) or $E=k_{+} S^{+} \oplus k_{-} S^{-}$(if $m$ is even).

Remark 6.2. The action $\phi$ is defined by its restriction to $\mathfrak{m} \otimes \mathfrak{m}$, which generates $\operatorname{spin}(\mathfrak{m})$. This is given by $\phi(X \otimes Y)=-\sigma(X) \overline{\sigma(Y)}^{t}$.

As an application of Lemma 6.1, we have the following classification of these operators:

Theorem 6.3. a. Let $D$ be an invariant elliptic first order differential operator on a homogeneous bundle over a spin symmetric space. Then, D is a twisted Dirac operator, plus a bundle map. 
b. Furthermore, if $\theta D=-D \theta$, where $\theta$ is the reflection from the symmetric structure, then $D$ is a twisted Dirac operator without the addition of a bundle map.

Proof. a. The proof of this is the same as the proof of Theorem 4.2 in [7]. b. Let $\phi$ be a bundle map such that $\theta \phi=-\phi \theta$. Then to prove part b we must show that $\phi=0$. Since $\phi$ is an even (zero) order map, we have $\theta \phi=\phi \theta$. Thus, $\theta \phi=\phi \theta=0$ and so $\phi=0$.

Remark. If $D$ is a twisted Dirac operator defined by using the Levi-Civita connection, then $\theta D=-D \theta$. If $D_{1}$ is a similar operator defined using a different connection, then $D-D_{1}$ is a bundle map. Thus, Theorem $6.3 \mathrm{~b}$ establishes the uniqueness of the Dirac operator which satisfies $\theta D=-D \theta$.

\section{First Order Elliptic Invariant Operators on a Riemannian Manifold.}

Let $M$ be a Riemannian manifold with frame bundle $F$. This has structure group $O(n)(S O(n)$ if $M$ is orientable) where $n=\operatorname{dim} M$. If $\pi: O(n) \rightarrow$ Aut $E$ is a representation, then the associated bundle is $\mathbf{E}=F \times_{\pi} E$. Let $J^{1}(\mathbf{E})$ be the first jet bundle. Then the action of $O(n)$ on $E$ extends to an action on $J^{1}(\mathbf{E})$. An invariant first order differential operator is then defined to be an $O(n)$-equivariant map:

$$
D: J^{1}(\mathbf{E}) \rightarrow \mathbf{E} .
$$

Now consider the jet bundle exact sequence:

$$
0 \rightarrow T \otimes \mathbf{E} \stackrel{i}{\rightarrow} J^{1}(\mathbf{E}) \stackrel{P}{\rightarrow} \mathbf{E} \rightarrow 0,
$$

where $T=T(M)$ is the tangent bundle of $M$. The symbol of $D$ is then the composition

$$
\sigma=D \circ i \text {. }
$$

Now we restrict to the case $n \geq 3$. Since $O(n)$ is compact and has trivial prologation the exact sequence (7.2) has a splitting by an $O(n)$ invariant map $s: \mathbf{E} \rightarrow J^{1}(\mathbf{E})$. Then $D$ gives rise to a bundle map $\phi=D \circ s: \mathbf{E} \rightarrow \mathbf{E}$. Thus, we can write $D$ as the sum of a symbol map and a bundle map:

$$
D=\sigma+\phi,
$$

where all these maps are $O(n)$ invariant and we have abused the notation by omitting the implied maps $p$ and the splitting.

Now $M$ is uniformly 1-flat and so the question of classifying first order operators is the same for all manifolds. Thus, it is sufficient to consider the sphere $S^{n}$. Since $S^{n} \cong \operatorname{Spin}(n+1) / \operatorname{Spin}(n)$ then, for $n \geq 3$, we can use Theorem 6.3 to obtain the result more generally. Note that by replacing $M$ with a suitable cover we can obtain a spin manifold. 
Theorem 7.1. Let $M$ be a compact Riemannian spin manifold of dimension $n \geq 3$. Then, if $D$ is an invariant elliptic operator on a bundle associated to a representation of Spin $(n), D$ is a twisted Dirac operator plus a bundle map.

\section{Appendix: The Example of $S U(2)$.}

Let $V_{n}$ be the irreducible representation of dimension $n$ with $\pi_{n}: S U(2) \rightarrow$ Aut $V_{n}$. Then $S=V_{2}$ and $\chi=\pi_{2}$. Rather than using normal coordinates on $S U(2) \times S U(2) / S U(2)$ we use left translation to trivialize the spin bundle:

$$
\Gamma(\mathbf{S})=C^{\infty}(S U(2)) \otimes S=\sum_{n=1}^{\infty} V_{n} \otimes V_{n}^{*} \otimes S .
$$

In this case $V_{n}^{*}=V_{n}$ since the representations are self dual for $S U(2)$. The various group actions are

$$
\begin{gathered}
\text { on the left } \ell(g)=\nu(g) \otimes 1 \otimes 1=\sum \pi_{n}(g) \otimes 1 \otimes 1 \\
\text { on the right } r(g)=1 \otimes \nu(g) \otimes \chi=\sum 1 \otimes \pi_{n}(g) \otimes \chi(g) .
\end{gathered}
$$

Now $V_{n} \otimes S=V_{n+1} \oplus V_{n-1}$. Thus, the isotypic components for first the left and then the right translation are

$$
V_{n} \otimes V_{n}^{*} \otimes S=V_{n} \otimes V_{n+1} \oplus V_{n} \otimes V_{n-1} .
$$

These are invariant spaces for the Dirac Operator $P$ and from [11] we see these are in fact the eigenspaces of $P$ :

$$
\begin{aligned}
& V_{n} \otimes V_{n+1} \quad \text { with eigenvalue } n+1 / 2 \\
& V_{n} \otimes V_{n-1} \text { with eigenvalue }-n+1 / 2 \text {. }
\end{aligned}
$$

The inversion map, $i(x)=x^{-1}$, acts on these by

$$
i: V_{n} \otimes V_{n+1} \rightarrow V_{n+1} \otimes V_{n} .
$$

This intertwines $P$ and $-P$ : Interchanging eigenvalue $n-1 / 2$ on $V_{n-1} \otimes V_{n}$ with $-n+1 / 2$ on $V_{n} \otimes V_{n-1}$. Clearly, we have spectral symmetry for $P$, but no $S U(2)$-equivariant symmetry for either the left or the right action.

If we regard $S U(2)$ as a symmetric space $S U(2)=S U(2) \times S U(2) / H$, where $H \cong S U(2)$ as the diagonal subgroup, then the left action is by $S U(2) \times 1$ and the right action by $1 \times S U(2)$. The group $H$ acts by the adjoint action. Thus the eigenspaces $V_{n-1} \otimes V_{n}$ and $V_{n} \otimes V_{n-1}$ are $H$-equivariant and the Dirac operator has equivariant symmetry for the adjoint action.

We apply this result to obtain the vanishing of the $\widehat{A}$-genus for homogeneous spin manifold $G / H$ of dimension $4 k$ with $\operatorname{rank} H<\operatorname{rank} G$. Let $M=G / H \times S U(2) /\{ \pm 1\}$. Then the space of spinors on $M$ decomposes

$$
\Delta=\Delta_{X}^{+} \otimes \Delta_{Y} \oplus \Delta_{X}^{-} \otimes \Delta_{Y},
$$


where we write $X$ for $G / H$ and $Y$ for $S U(2) /\{ \pm 1\}$. Using this decomposition, a direct calculuation yields:

Lemma A.1. $\eta_{M}(S)=\widehat{A}(X) \eta_{Y}(S)$.

Proof. This follows from the corresponding decomposition for the Dirac operator: $P_{M}=P_{X} \otimes \omega_{Y}+\omega_{X} \otimes P_{Y}$, together with the observation that $\Delta_{X}^{+}=\operatorname{ker} P_{X} \underset{\lambda \neq 0}{\oplus} \operatorname{ker}\left(P_{X}^{2}-\lambda\right)$ with the sum over nonzero $\lambda$. We note that $\widehat{A}(X)$ is the index of the operator $P_{X}$ restricted to map $\Delta_{X}^{+}$to $\Delta_{X}^{-}$.

To see $\widehat{A}(X)=0$ we observe that the results of [8] apply to $M$ so that $\eta_{M}(s)=0$. However, $\eta_{Y}(0) \neq 0$ and so $\widehat{A}(X)=0$.

Remark. We have identified $S U(2)=S^{3}$ from the introduction and made the specific choice $\Gamma=\{ \pm 1\}$ of discrete subgroup. Of course other choices are possible allowing consideration of the product $G / H \times S^{3} / \Gamma$ as discussed in the introduction.

\section{Appendix: Rank and Difference Rank.}

Let $M=G \backslash H$ then the rank of $M$ is the dimension of the maximal flat totally geodesic submanifold of $M$. The difference rank of $M$ is Rank $G$ Rank $H$.

Lemma B.1. Rank $M \geq$ Difference rank $M$.

If rank $M=$ difference rank $M$ then $M$ has split rank. The cases when $M$ has split rank are:

(1) If $M=K \times K / K$, these are spaces of type II.

(2) $S U(2 n) / S p(n)$ where rank is $n-1$.

(3) $E_{6} / F_{4}$ where rank is 2 .

These results come from the classification in [9].

\section{References}

[1] J.F. Adams, Lectures on Exception Lie Groups, (eds. Zafer Mahmud and Mamoru Mimura), Chicago Lectures in Mathematics, University of Chicago Press, Chicago, 1996.

[2] M.F. Atiyah, R. Bott and A. Shapiro, Clifford modules, Topology, 3(1) (1964), 3-38.

[3] M.F. Atiyah and F. Hirzebruch, Spin Manifolds and Group Actions, in Essays on Topology and Related Topics, Memoires dédiés ä Georges de Rham, Springer-Verlag, Berlin-Heidelberg-New York, (1970), 18-28.

[4] M.F. Atiyah, V.K. Patodi and I.M. Singer, Spectral asymmetry and Riemannian geometry I, Proc. Camb. Phil. Soc., 77 (1975), 43-69.

[5] M.F. Atiyah and W. Schmid, A geometric construction of the discrete series for semisimple Lie groups, Invent. Math., 42 (1977), 1-62. 
[6] H.D. Fegan, The spectrum of the Laplacian on forms over a Lie group, Pac. J. Math., 90 (1980), 373-387.

[7] H.D. Fegan and B. Steer, First order operators on manifolds with a group action, Can. J. Math., 48 (1996), 758-776.

[8] - Spectral symmetry of the Dirac operators in the presence of a group action, Trans. AMS, 335 (1993), 631-647.

[9] G. Helgason, Differential Geometry, Lie Groups and Symmetric Spaces, Academic Press, New York, 1978.

[10] F. Hirzebruch and P. Slodowy, Elliptic genera, involutions and homogeneous spin manifolds, Geom. Dedicata, 35 (1990), 3090-343.

[11] N. Hitchin, Harmonic spinors, Advances in Math., 14 (1974), 1-55.

[12] J. Millson, Closed geodesics and the Eta invariant, Ann. Math., 108 (1978), 1-39.

[13] H. Moscovici and R. Stanton, Eta invariants of Dirac operators on locally symmetric manifolds, Invent. Math., 95 (1989), 629-666.

[14] R. Parthasarathy, Dirac operators and the discrete series, Ann. of Math., 96 (1972), 1-30.

[15] J. Seade and B. Steer, A note on the Eta function for quotients of $P S L_{2}(\mathbb{R})$ by co-compact Fuchsian groups, Topology, 26 (1987), 79-91.

[16] G. Shimura, Invariant differential operators on Hermitian symmetric spaces, Ann. Math., 132 (1990), 237-272.

Received April 2, 1998 and revised June 23, 1999.

LEHIGH UNIVERSITY

Bethlehem, PA 18015-3174

E-mail address: hdf3@lehigh.edu

Hertford College

OXFORD OX1 3BW

ENGLAND

UNIVERSITY OF HELSINKI

HALLITUSKATU 15

FinlAND 00014 